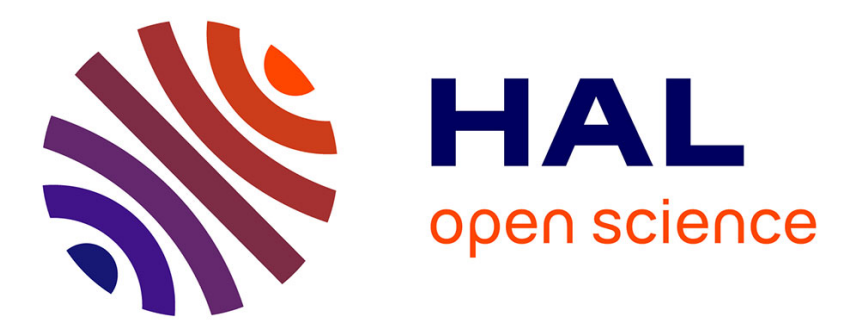

\title{
Invariant Unscented Kalman Filter with application to attitude estimation
}

Jean-Philippe Condomines, Cédric Seren, Gautier Hattenberger

\section{To cite this version:}

Jean-Philippe Condomines, Cédric Seren, Gautier Hattenberger. Invariant Unscented Kalman Filter with application to attitude estimation. CDC 2017, 56th IEEE conference on decision and control, Dec 2017, Melbourne, Australia. pp.ISBN: 978-1-5090-2874-0, 10.1109/CDC.2017.8264063 . hal01509884

\section{HAL Id: hal-01509884 \\ https://hal-enac.archives-ouvertes.fr/hal-01509884}

Submitted on 8 Jun 2017

HAL is a multi-disciplinary open access archive for the deposit and dissemination of scientific research documents, whether they are published or not. The documents may come from teaching and research institutions in France or abroad, or from public or private research centers.
L'archive ouverte pluridisciplinaire HAL, est destinée au dépôt et à la diffusion de documents scientifiques de niveau recherche, publiés ou non, émanant des établissements d'enseignement et de recherche français ou étrangers, des laboratoires publics ou privés. 


\title{
Invariant Unscented Kalman Filter with application to attitude estimation
}

\author{
Jean-Philippe Condomines ${ }^{a}$, Cédric Seren $^{b}$ and Gautier Hattenberger ${ }^{c}$
}

\begin{abstract}
The Invariant UKF, named IUKF, is a recently introduced algorithm dedicated to nonlinear systems possessing symmetries as illustrated by the quaternion-based kinematics modeling of a mini-UAV (Unmanned Aircraft Vehicle) considered in this paper. Within an invariant framework, this algorithm suggests a systematic approach to determine all the symmetry-preserving terms, without requiring any compatibility condition such as proposed in the $\pi$-IUKF, by introducing both notion of invariant output errors and UKF algorithm formulation. We propose in this paper to evaluate the applicability of our proposed IUKF observer to the case of attitude estimation for small UAVs using low-cost sensors. The IUKF algorithm is successfully validated in experiments and demonstrates that nonlinear state estimation converges on a much bigger set of trajectories than for more traditional approaches.
\end{abstract}

\section{INTRODUCTION}

Many recent progresses in the miniaturization of electromechanical sensors have led to the design of small and cheap integrated navigation system hardwares (complete IMU: Inertial Measurement Unit, GPS : Global Positioning System module, etc.), which have, for their part, contributed to boost significantly the market of mini-UAVs (Unmanned Air Vehicles) over the last decades, making them more accessible to everyone. Nevertheless, this accessibility is frequently inconsistent with good measurement performances. For instance, the GPS modules commonly used with the Paparazzi $i^{1}$ autopilot deliver an absolute position with an average accuracy of 5 meters, up to $10 \mathrm{~m}$ under certain flight conditions. Therefore, a need for multiple microelectromechanical sensors (MEMS) data fusion arises, especially when the final objective consists in developing robust and powerful advanced control strategies for mini-UAVs that can be viewed as complex autonomous electromechanical system. So much so that full state (or estimated state) feedback designs (cf. LQG/LTR syntheses) provide full authority to control efficiently in terms of stability and performances UAVs for accomplishing various missions. To this aim, nonlinear estimation offers several well-proven algorithmic techniques which permit to recover an acceptable level of accuracy on some key flight parameters (anemometric angles, orientation/attitude, linear and angular speeds, position, etc.) for mini-UAVs closed-loop handling qualities.

\footnotetext{
${ }^{a}$ Assistant-professor at ENAC, University of Toulouse, Toulouse, France jean-philippe.condomineserecherche.enac.fr

${ }^{b}$ Researcher at the Department of Control Systems and Flight Dynamics, ONERA-The French Aerospace Lab, Toulouse, France cedric.seren@onera.fr

${ }^{c}$ Assistant-professor at ENAC, University of Toulouse, Toulouse, France gautier.hattenbergerdenac.fr

${ }^{1}$ https://paparazziuav.org/
}

An overview of nonlinear estimation methods can be found in the literature from many surveys or books (e.g.,[13],[14]). Figure 1 attempts to propose a classification of these latter and positions the article's topic in it (white terms in grey boxes). As they merge different nonlinear estimation principles, Kalman-based invariant observers can be qualified as hybrid filters. Although dynamical systems possessing symmetries have been studied in control theory, few results taking benefit of system invariances for observers design exist today. Invariant nonlinear estimation theory appears so as a young research area in which the first main contributions can be dated from the beginning of 2000s ([1], [3], [2], [5], [4], [6], [8], [9], [15], [10], [11], [17], [18]).

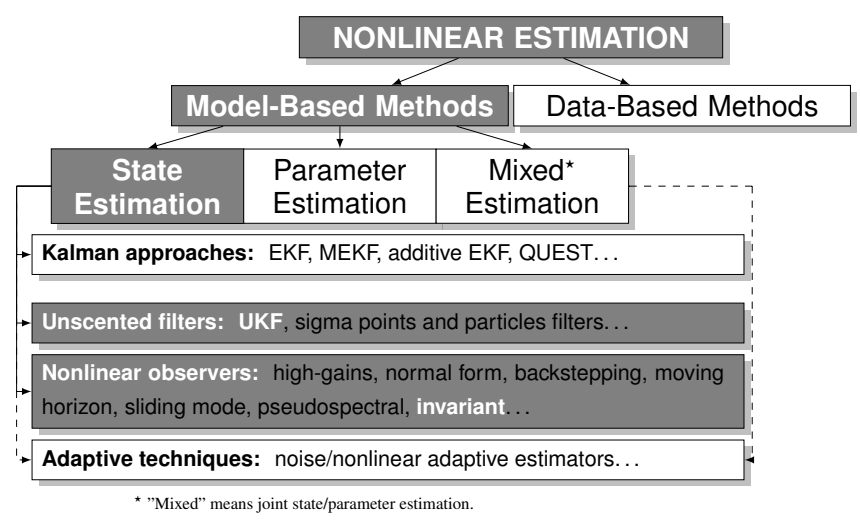

Fig. 1. Classification of existing nonlinear state estimation techniques and paper topic positioning.

Initially, research was going on in the development of constructive methods to derive invariant observers for nonlinear estimation purposes which preserve systems' symmetries. If this kind of non-systematic approaches keeps physical readiness, it requires however to tune an important number of setting parameters potentially when computing estimation gains, which can be cumbersome for complex system modelings. That is why, researchers have then tried to develop more systematic techniques which are able to facilitate estimators' gains computation. There exist two major approaches to perform Bayesian filtering for a state evolving on an Euclidean space : the Kalman filter (KF) such as (Extended Kalman filter or Unscented Kalman filter) and the particle filters. However among those methods only a few works tried to extend them to manifolds. The Invariant Extended Kalman Filter (IEKF - cf. bibliographical references [8], [4]) permits to determine gain matrices for minimum variance estimation. This optimality must be considered here w.r.t. an invariant state estimation error which 
will be defined precisely further. An important drawback in this method is that it requires to linearize a system of differential equations which govern the invariant state estimation error dynamics. Such an operation appears suitable for simple system modelings only s.t. UAVs whose dynamics can be represented easily based on kinematics relationships. Indeed, this kind of nonlinear state space representation can be differentiated analytically towards its state vector. For more complex system modelings, this linearization may be difficult to carry out. Nevertheless, the IEKF, and more generally invariant observers, are characterized by a larger convergence domain, due to the exploitation of systems' symmetries within the estimation algorithm (i.e., within filter equations and gains computation), and present very good performances in practice. In order to derive more tractable nonlinear invariant state estimation algorithms, motivated by the practical problems encountered by the authors with miniUAVs flight control and guidance, civil Aircraft modeling and identification and dynamic system fault detection, isolation and recovery, an hybridization of the Unscented KF (UKF) principles [20], [16], [19] with invariant observers theory has been recently proposed in [10], [11]. Among other things, it has been proved in these bibliographical references that an Invariant UKF-like estimator (named IUKF) could be simply designed by introducing both notions of invariant state estimation and invariant output errors within any UKF algorithm formulation, whatever this latter corresponds to the standard version of the algorithm or to some square-root/UD factorized ones. This article focuses on these recent research works and details the theoretical aspects in the sequel.

In the sequel, §II presents the theoretical background of our proposed IUKF estimation algorithm and an illustrative example fitted out with a tilt sensor system. $\S I I I$ gathers all the results obtained after solving the Attitude and Heading Reference System (AHRS) estimation problem in real conditions.

\section{The InVAriant Unscented Kalman Filter}

Inspired by the theory of continuous-time symmetry preserving observer [7] a novel and original UKF-based approach has been developed in [12] to address the approximation issue of the invariant EKF without requiring any linearization of the dynamical systems equations or compatibility condition such as proposed in the $\pi$-IUKF algorithm [10], [11]. The IUKF relies on the basic theoretical principles developed by Julier and Uhlmann at the beginning of 2000s (see [16]) which have been since widely applied to various nonlinear state estimation problems (cf. [19]).

Proposition 1: Considering a whole state space representation, the composite transformation $\phi_{\mathbf{g} \in \mathrm{G}}=\left(\psi_{\mathbf{g}}, \varphi_{\mathbf{g}}, \rho_{\mathbf{g}}\right)$ and starting from initial values $\hat{\mathbf{x}}_{0}=\mathrm{E}\left[\mathbf{x}_{0}\right], \mathbf{P}_{0}^{\mathbf{x x}}=$ $\mathrm{E}\left[\boldsymbol{\eta}\left(\mathbf{x}_{0}, \hat{\mathbf{x}}_{0}\right) \boldsymbol{\eta}^{T}\left(\mathbf{x}_{0}, \hat{\mathbf{x}}_{0}\right)\right]$ the two-steps procedure (prediction/correction) permit to design the following invariant nonlinear state observer in discrete time:

$$
\begin{aligned}
& \text { ( } \forall i \in \llbracket 0 ; 2 n \rrbracket \text {, } \\
& \boldsymbol{\mathcal { X }}_{k+1 \mid k}^{(i)}=f_{d}\left(\boldsymbol{\mathcal { X }}_{k \mid k}^{(i)}, \mathbf{u}_{k}\right) \Rightarrow \hat{\mathbf{x}}_{k+1 \mid k}=\sum_{i=0}^{2 n} W_{m}^{(i)} \boldsymbol{\mathcal { X }}_{k+1 \mid k}^{(i)} \\
& \text { (2) } \mathbf{S}_{k+1 \mid k}^{\mathbf{x x}}= \\
& \operatorname{qr}\left[\sqrt { W _ { c } ^ { ( 1 ) } } \left(\boldsymbol{\eta}\left(\hat{\mathbf{x}}_{k+1 \mid k}, \boldsymbol{\mathcal { X }}_{k+1 \mid k}^{(1)}\right) \cdot \ldots\right.\right. \\
& \left.\left\{\boldsymbol{\eta}\left(\hat{\mathbf{x}}_{k+1 \mid k}, \boldsymbol{\mathcal { X }}_{k+1 \mid k}^{(2 n)}\right)\right) \mathbf{V}_{k}^{1 / 2}\right] \\
& \text { cholupdate }\left(\mathbf{S}_{k+1 \mid k}^{\mathbf{x x}}, \boldsymbol{\eta}\left(\hat{\mathbf{x}}_{k+1 \mid k}, \boldsymbol{\mathcal { X }}_{k+1 \mid k}^{(0)}\right), W_{c}^{(0)}\right) \\
& \text { (3) } \forall i \in \llbracket 0 ; 2 n \rrbracket \text {, } \\
& \hat{\mathbf{y}}_{k+1 \mid k}^{(i)}=h_{d}\left(\boldsymbol{\mathcal { X }}_{k \mid k}^{(i)}, \mathbf{u}_{k}\right) \Rightarrow \hat{\mathbf{y}}_{k+1 \mid k}=\sum_{i=0}^{2 n} W_{m}^{(i)} \hat{\mathbf{y}}_{k+1 \mid k}^{(i)}
\end{aligned}
$$

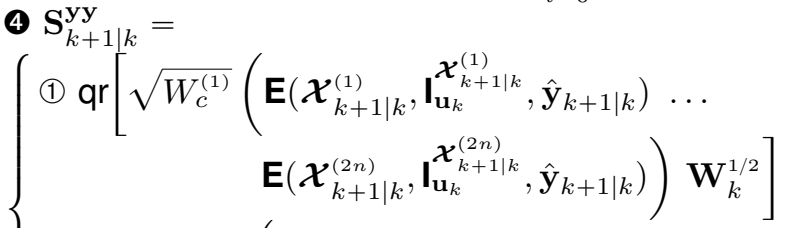

$$
\begin{aligned}
& \text { (2) cholupdate }\left(\mathbf{S}_{k+1 \mid k}^{\mathbf{y y}}, \ldots\right. \\
& \left.\mathbf{E}\left(\mathcal{X}_{k+1 \mid k}^{(0)}, \mathbf{l}_{\mathbf{u}_{k}}^{\boldsymbol{X}_{k+1 \mid k}^{(0)}}, \hat{\mathbf{y}}_{k+1 \mid k}\right), W_{c}^{(0)}\right) \\
& \text { (6) } \mathbf{P}_{k+1 \mid k}^{\mathrm{xy}}= \\
& \sum_{i=0}^{2 n} W_{c}^{(i)} \boldsymbol{\eta}\left(\boldsymbol{\mathcal { X }}_{k+1 \mid k}^{(i)}, \hat{\mathbf{x}}_{k+1 \mid k}\right) \mathbf{E}^{T}\left(\boldsymbol{\mathcal { X }}_{k+1 \mid k}^{(i)}, \mathbf{l}_{\mathbf{u}_{k}}^{\boldsymbol{X}_{k+1 \mid k}^{(i)}}, \hat{\mathbf{y}}_{k+1 \mid k}\right) \\
& \text { (6 } \forall i \in \llbracket 1 ; n \rrbracket \text {, } \\
& \overline{\mathbf{K}}_{i}[\mathbf{E}]=i^{\text {th }} \text { row of } \mathbf{K}=\left(\mathbf{P}_{k+1 \mid k}^{\mathbf{x y}} /\left(\mathbf{S}_{k+1 \mid k}^{\mathbf{y y}}\right)^{T}\right) / \mathbf{S}_{k+1 \mid k}^{\mathbf{y y}} \\
& \left(\boldsymbol{F} F: \hat{\mathbf{x}}_{k+1 \mid k+1}=\right. \\
& \hat{\mathbf{x}}_{k+1 \mid k}+\sum_{i=1}^{n} \overline{\mathbf{K}}_{i}[\mathbf{E}] \cdot \mathbf{E}\left(\hat{\mathbf{x}}_{k+1 \mid k},{ }_{\mathbf{u}_{k}}^{\hat{\mathbf{x}}_{k+1 \mid k}}, \mathbf{z}_{k+1}\right) \cdot \mathbf{w}_{i}\left(\hat{\mathbf{x}}_{k+1 \mid k}\right) \\
& \boldsymbol{8} \mathbf{S}_{k+1 \mid k+1}^{\mathbf{x x}}=\text { cholupdate }\left(\mathbf{S}_{k+1 \mid k}^{\mathbf{x x}}, \mathbf{K} \mathbf{S}_{k+1 \mid k}^{\mathbf{y y}},-1\right)
\end{aligned}
$$

Previous matricial computations rely on both $Q R$ decomposition and rank 1 update to Cholesky factorization (cholupdate). Local transformations $\left(\psi_{\mathbf{g}}, \varphi_{\mathbf{g}}, \rho_{\mathbf{g}}\right)$ are here defined as for a dynamical system preserving symmetries [7]. In this formulation, state, output and crossed error covariances are now defined from system modeling invariants. It is clear by transitivity that these matricial quantities are left unchanged by the composite transformation $\phi_{\mathbf{g} \in \mathrm{G}}=$ $\left(\psi_{\mathbf{g}}, \varphi_{\mathbf{g}}, \rho_{\mathbf{g}}\right)$. Unlike the Invariant Extended Kalman Filter (IEKF), the proposed IUKF does not require a linearization of $\dot{\boldsymbol{\eta}}\left(\mathrm{x}_{t}, \hat{\mathbf{x}}_{t}\right)$ w.r.t $\boldsymbol{\eta}$ for its gain matrix computation step. When any given permanent trajectory $t \mapsto\left(\mathbf{x}_{p}(t), \mathbf{u}_{p}(t)\right)$ is followed (i.e., s.t. $\left.\forall t, \mathbf{I}_{\mathbf{u}_{p}}^{\mathbf{x}_{p}}(t)=\overline{\mathbf{I}}\right), 1^{\text {st }}$ order approximation of the dynamical error $\boldsymbol{\eta}$ shows that if $\mathbf{K}$ is also determined s.t. matrix $\partial \Upsilon(0, \overline{\mathbf{I}}) / \partial \boldsymbol{\eta}$ is stable, then observer $F$ will converge locally around $\left(\mathbf{x}_{p}(t), \mathbf{u}_{p}(t)\right)$. Reuse of system modeling invariances within invariant observer design also guarantees that it will converge for any group action image $\left(\psi_{\mathbf{g}}\left(\mathbf{u}_{p}(t)\right), \varphi_{\mathbf{g}}\left(\mathbf{x}_{p}(t)\right)\right)_{\mathbf{g} \in \mathbf{G}}$. This property is remarkable especially for dynamical systems described by kinematics relationships whose dynamics is invariant by translation and 
rotation movements inside an invariant frame. By doing this, correction step procedure relies on the determination of the $n$ additive gain which depend on system fundamental invariants and invariant innovation terms. Moreover, the invariant correction terms are projected on each component of the dynamical equations by considering the canonical basis of $\mathbb{R}^{n}$ such as $\mathcal{B}\left(\hat{\mathbf{x}}_{k+1 \mid k}\right)=\left\{\omega_{i}\left(\hat{\mathbf{x}}_{k+1 \mid k}\right)\right\}_{i \in \llbracket 1 ; n \rrbracket}$ vectors form an invariant frame for each $\hat{\mathbf{x}} \in \mathcal{X}$. Thus, the IUKF algorithm relies on a multiple parametrization defined by local transformation groups. Considering the transformation group $\phi_{g}=\left(\varphi_{g}, \psi_{g}, \rho_{g}\right)$ each inverse of sigma point can be defined as a local parameter of $(2 n+1)$ invariant frame which project each sigma point on the neutral element $e$ thought the local application $\varphi_{g}$.

\section{BENCHMARK AND APPLICATION ON ATTITUDE ESTIMATION}

\section{A. Dynamic system modeling}

This subsection details the generic modeling used to tackle and solve the issue of estimating some key flight variables (attitude-orientation, angle rates, etc.) of mini-UAVs fitted out with an Attitude and Heading Reference System (AHRS). UAVs dynamics representation corresponds here to a pure quaternionial kinematics modeling (whose related quaternion will be denoted by $\mathbf{q}$ ), supplemented by additive state variables which represent low frequency sensors' imperfections (such as slowly varying biases denoted by $a_{s}$ and $b_{s}$ ). Thereby, we consider:

$$
\Sigma:\left\{\begin{array}{c}
\dot{\mathbf{x}}=\left(\begin{array}{l}
\dot{\mathbf{q}}=\mathbf{q} *\left(\boldsymbol{\omega}_{m}-\boldsymbol{\omega}_{b}\right) / 2 \\
\dot{\boldsymbol{\omega}}_{b}=\mathbf{0} \\
\dot{a}_{s}=0 \\
\dot{b}_{s}=0
\end{array}\right), \\
\mathbf{y}=\left(\begin{array}{l}
\mathbf{y}_{\mathbf{A}}=a_{s} \mathbf{q}^{-1} * \mathbf{A} * \mathbf{q} \\
\mathbf{y}_{\mathbf{B}}=b_{s} \mathbf{q}^{-1} * \mathbf{B} * \mathbf{q}
\end{array}\right)
\end{array}\right.
$$

where $\boldsymbol{\omega}_{m}$ is seen as an imperfect and noisy, but known, measured input, like B. Constant $\mathbf{A}=\left(\begin{array}{lll}0 & 0 & g\end{array}\right)^{T}$ refers to the local Earth's gravity vector. Nonlinear state space representation of Eq. (1) can be described in a compact form s.t. $\dot{\mathbf{x}}=f(\mathbf{x}, \mathbf{u})$ and $\mathbf{y}=h(\mathbf{x}, \mathbf{u})$ where $\mathbf{u}=\boldsymbol{\omega}_{m}$, $\mathbf{x}=\left(\begin{array}{llll}\mathbf{q}^{T} & \boldsymbol{\omega}_{b}^{T} & a_{s} & b_{s}\end{array}\right)^{T}$ and $\mathbf{y}=\left(\begin{array}{ll}\mathbf{y}_{\mathbf{A}}^{T} & \mathbf{y}_{\mathbf{B}}^{T}\end{array}\right)^{T}$ are the input, state and output vectors respectively. The nonlinear state estimation problem makes use of 3 triaxial sensors which deliver a total of 9 scalar measurement signals: 3 magnetometers permit to obtain a local measurement of Earth's magnetic field, which is known constant and expressed in the body-fixed frame s.t. vector $\mathbf{y}_{\mathbf{B}}=\mathbf{q}^{-1} * \mathbf{B} * \mathbf{q}$ (where $\left.\mathbf{B}=\left(B_{x} B_{y} B_{z}\right)^{T}\right)$ can be considered as an output of the observation equations; 3 gyroscopes produce the measurements associated with the instantaneous angular rates gathered in $\boldsymbol{\omega}_{m} \in \mathbb{R}^{3}$ s.t. $\boldsymbol{\omega}_{m}=\left(\begin{array}{lll}\omega_{m x} & \omega_{m y} & \omega_{m z}\end{array}\right)^{T}$; and 3 accelerometers provide the measured output signals corresponding to the specific acceleration, denoted by $\mathbf{a}_{m} \in$ $\mathbb{R}^{3}$ with $\mathbf{a}_{m}=\left(a_{m x} a_{m y} a_{m z}\right)^{T}$. As no velocity and position informations are available (no GPS, nor airspeed sensor), this AHRS is often qualified as non-aided.

\section{B. IUKF estimator derivation}

Let's consider the observer in [11] for the IUKF algorithm s.t.:

$$
\begin{aligned}
\dot{\hat{\mathbf{q}}}= & \frac{\hat{\mathbf{q}} *\left(\boldsymbol{\omega}_{m}-\hat{\boldsymbol{\omega}}_{b}\right)}{2}+\ldots \\
& \sum_{i=1}^{3}\left(\overline{\mathbf{K}}_{i}^{1: 3}[\mathbf{E}] \cdot \mathbf{E}_{\mathbf{A}}+\overline{\mathbf{K}}_{i}^{4: 6}[\mathbf{E}] \cdot \mathbf{E}_{\mathbf{B}}\right) \boldsymbol{v}_{i} * \hat{\mathbf{q}}+\mathbf{C}_{\hat{\mathbf{q}}} \\
\dot{\hat{\boldsymbol{\omega}}}_{b}= & \hat{\mathbf{q}}^{-1} *\left(\sum_{i=4}^{6}\left(\overline{\mathbf{K}}_{i}^{1: 3}[\mathbf{E}] \cdot \mathbf{E}_{\mathbf{A}}+\overline{\mathbf{K}}_{i}^{4: 6}[\mathbf{E}] \cdot \mathbf{E}_{\mathbf{B}}\right)\right) * \hat{\mathbf{q}} \\
\dot{\hat{a}}_{s}= & \hat{a}_{s} \cdot\left(\overline{\mathbf{K}}_{7}^{1: 3}[\mathbf{E}] \cdot \mathbf{E}_{\mathbf{A}}+\overline{\mathbf{K}}_{7}^{4: 6}[\mathbf{E}] \cdot \mathbf{E}_{\mathbf{B}}\right) \\
\dot{\hat{b}}_{s} & =\hat{b}_{s} \cdot\left(\overline{\mathbf{K}}_{8}^{1: 3}[\mathbf{E}] \cdot \mathbf{E}_{\mathbf{A}}+\overline{\mathbf{K}}_{8}^{4: 6}[\mathbf{E}] \cdot \mathbf{E}_{\mathbf{B}}\right)
\end{aligned}
$$

The invariant state estimation error dynamics $\dot{\boldsymbol{\eta}}\left(\mathrm{x}_{t}, \hat{\mathbf{x}}_{t}\right)$ is given by:

$$
\left\{\begin{array}{c}
\dot{\boldsymbol{\eta}}=\left(\sum_{i=1}^{3}\left(\overline{\mathbf{K}}_{i}^{1: 3}[\mathbf{E}] \cdot \mathbf{E}_{\mathbf{A}}+\overline{\mathbf{K}}_{i}^{4: 6}[\mathbf{E}] \cdot \mathbf{E}_{\mathbf{B}}\right) \boldsymbol{v}_{i}\right) * \boldsymbol{\eta}-\frac{1}{2} \boldsymbol{\eta} * \boldsymbol{\beta} \\
\dot{\boldsymbol{\beta}}=\left(\boldsymbol{\eta}^{-1} * \mathbf{I}_{\mathbf{u}}^{\hat{\mathbf{x}}} * \boldsymbol{\eta}\right) \times \boldsymbol{\beta}+\ldots \\
\boldsymbol{\eta}^{-1} * \sum_{i=1}^{3}\left(\overline{\mathbf{K}}_{i}^{1: 3}[\mathbf{E}] \cdot \mathbf{E}_{\mathbf{A}}+\overline{\mathbf{K}}_{i}^{4: 6}[\mathbf{E}] \cdot \mathbf{E}_{\mathbf{B}}\right) * \boldsymbol{\eta} \\
\dot{\boldsymbol{\alpha}}=-\boldsymbol{\alpha}\left(\overline{\mathbf{K}}_{7}^{1: 3}[\mathbf{E}] \cdot \mathbf{E}_{\mathbf{A}}+\overline{\mathbf{K}}_{7}^{4: 6}[\mathbf{E}] \cdot \mathbf{E}_{\mathbf{B}}\right) \\
\dot{\boldsymbol{\gamma}}=-\boldsymbol{\gamma}\left(\overline{\mathbf{K}}_{8}^{1: 3}[\mathbf{E}] \cdot \mathbf{E}_{\mathbf{A}}+\overline{\mathbf{K}}_{8}^{4: 6}[\mathbf{E}] \cdot \mathbf{E}_{\mathbf{B}}\right)
\end{array}\right.
$$

As it was mentioned, the reader can notice that the invariant state estimation error dynamics depends on system's trajectory $t \mapsto\left(\mathbf{x}_{t}, \mathbf{u}_{t}\right)$ through the invariant quantity $\mathbf{I}_{\mathbf{u}}^{\hat{\mathbf{x}}}$ which is a major difference with most of nonlinear estimators. Unlike the Invariant Extended Kalman Filter (IEKF - see references [8]), the proposed IUKF does not require a linearization of $\dot{\boldsymbol{\eta}}\left(\mathbf{x}_{t}, \hat{\mathbf{x}}_{t}\right)$ w.r.t. $\boldsymbol{\eta}$ for its gain matrix computation step. This linearization can appear as a difficult operation in itself and especially for any practical implementation.

\section{Experimental results}

We evaluate the IUKF performances experimentally by post-processing a set of experimental data on the basis of both the dynamical modeling of Eq. (1) and the filtering equations of Eq. (2). Figure 2 displays a picture of the Parrot

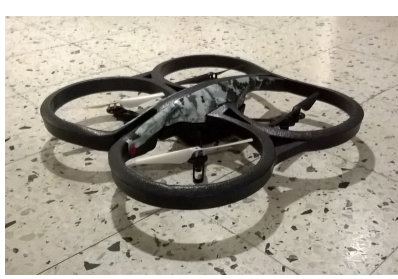

(a) Parrot quadrotor mini-UAV.

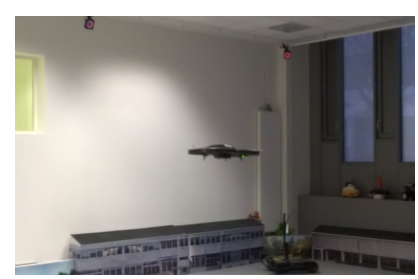

(b) Indoor flight experiment.
Fig. 2. Experimental materials: Parrot quadrotor mini-UAV and OptiTrack device.

quadrotor mini-UAV under test and an image of the indoor flight performed to gather these real data. It also illustrates that this experiment has been made using an OptiTrack system which permits to have at disposal absolute references (see http: //www.optitrack.com/). 

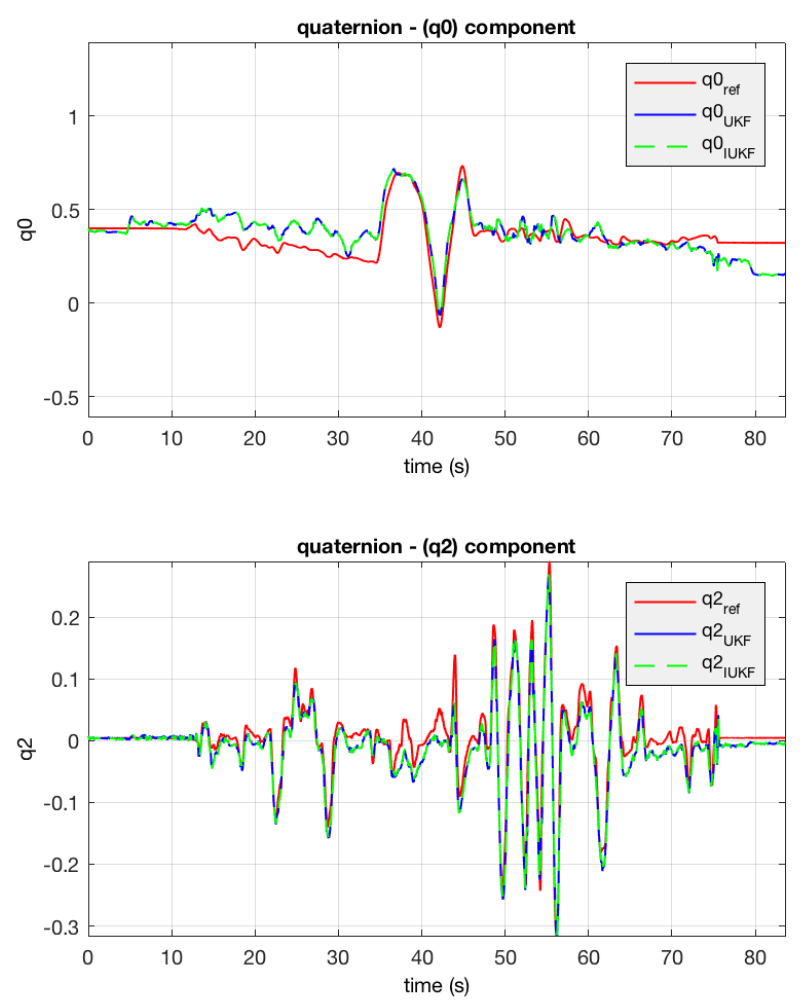
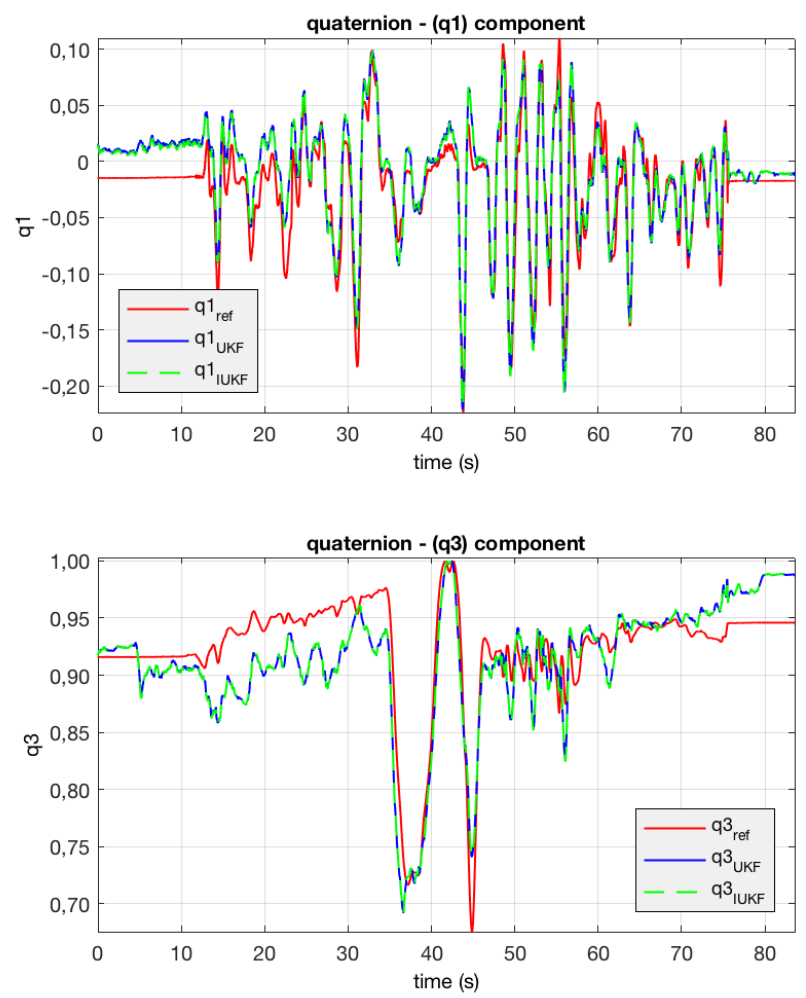

Fig. 3. Quaternion states estimation.

As no specific autopilot hardware device has been designed for this experiment, it is noteworthy that data fusion will merge low quality measurement signals delivered directly by the cheap MEMS sensors which equip any Parrot quadrotor. The interest of the following results relies less on the ability of the IUKF algorithm to estimate systems' states and outputs than on the practical verification of the theoretical properties asserted by the invariant observers framework when dealing with real data. To point out these latter, the results obtained with the IUKF algorithm have been systematically compared with the ones provided by a standard UKF approach. To lead a fair comparison, both techniques share identical setting parameters values i.e., similar estimated process and measurement covariances values for matrices $\mathbf{V}$ and $\mathbf{W}$. Figures 3 and 6 gather some estimation results due to a lack of space. Figure 3 shows the estimation results of the quaternion state components obtained by both UKF and IUKF algorithms. It is noticeable that both methods provide correct estimates w.r.t. the absolute references plotted in solid red lines. The differences between the two algorithms appear when we consider the dispersion around the estimated state trajectory. Indeed, the lines plotted on figure 5 , which correspond to the $\hat{\mathbf{q}}(t) \pm 3 \times \hat{\sigma}_{\mathbf{q}}(t)$ standard deviations around the mean estimated value, tend to prove that the IUKF estimation algorithm calculates more trustful quaternion estimates, or at least reduces the dispersion of these state estimates, due to the invariant framework used.

Based on these quaternion estimates, the instantaneous Euler attitude angles values, which describe at any time
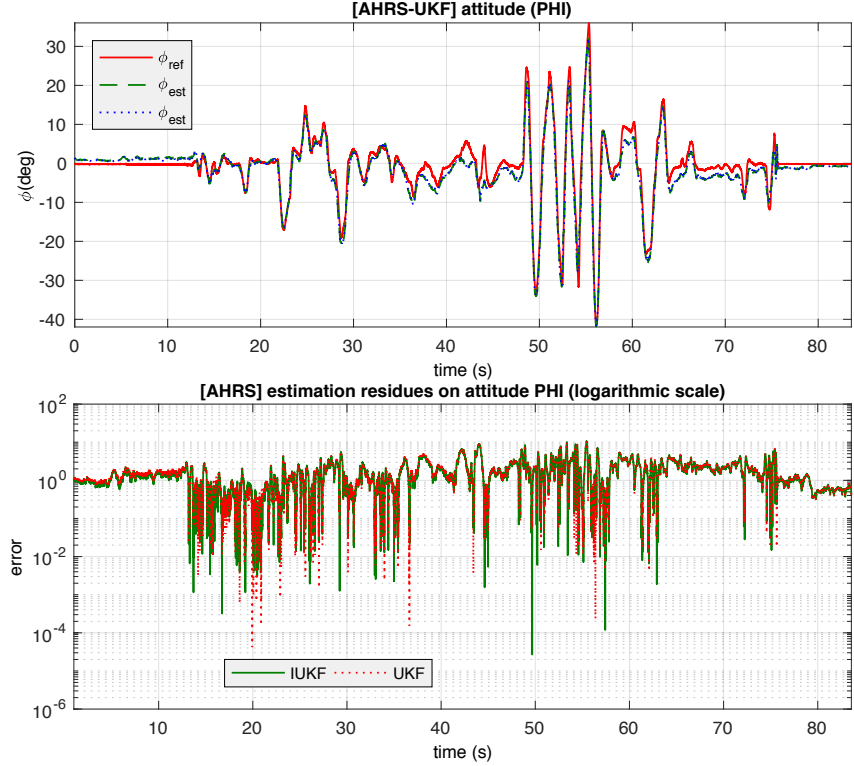

Fig. 4. Euler angles attitude estimation on $\phi$ and associated errors

instant the orientation of the flying Parrot, have been deduced through time and compared with the absolute references determined by the OptiTrack system. It appears that both algorithms allow to reconstruct a suitable attitude estimation for control purposes. Due to a lack of space only the $\phi$-axis estimation state error w.r.t. the absolute references is drawn in figure 4 using a logarithmic scale and show comparable results for both techniques. Expected differences 
[AHRS]

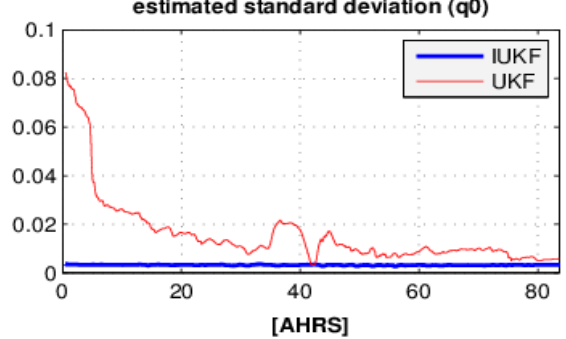

estimated standard deviation (q3)
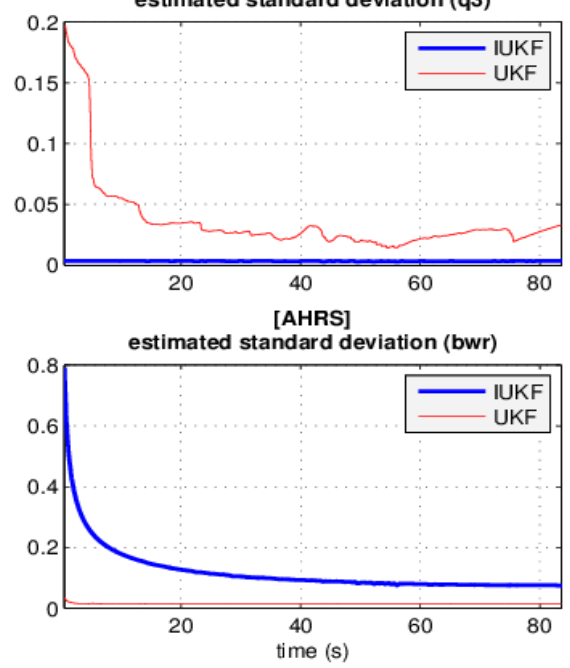

[AHRS]

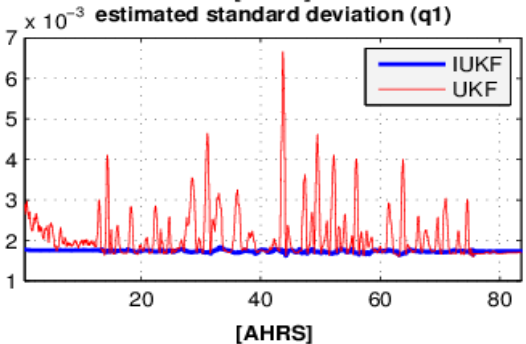

estimated standard deviation (bwp)
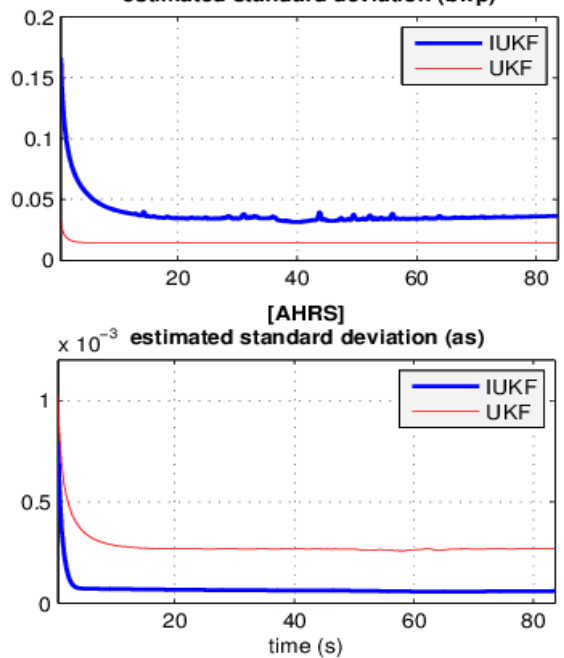

[AHRS]

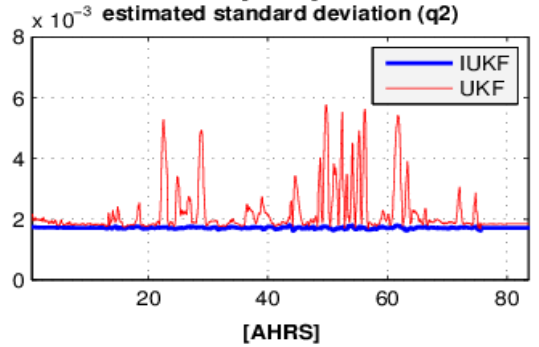

estimated standard deviation (bwq)
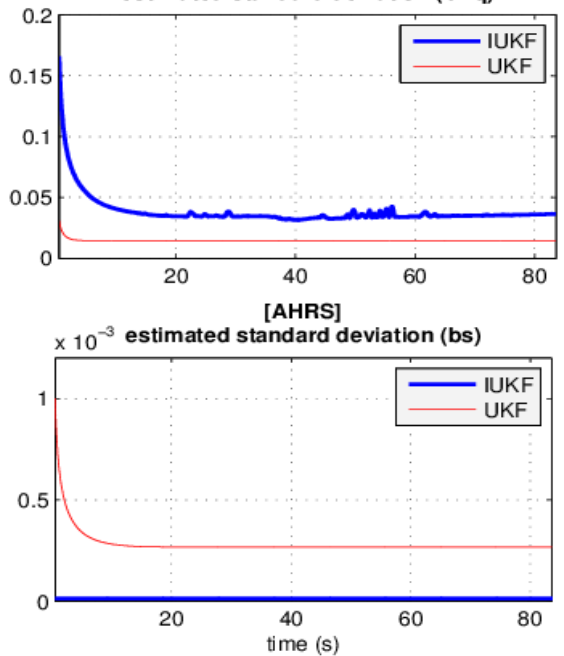

Fig. 5. Estimated theoretical standard deviations: comparison UKF/IUKF (state components).
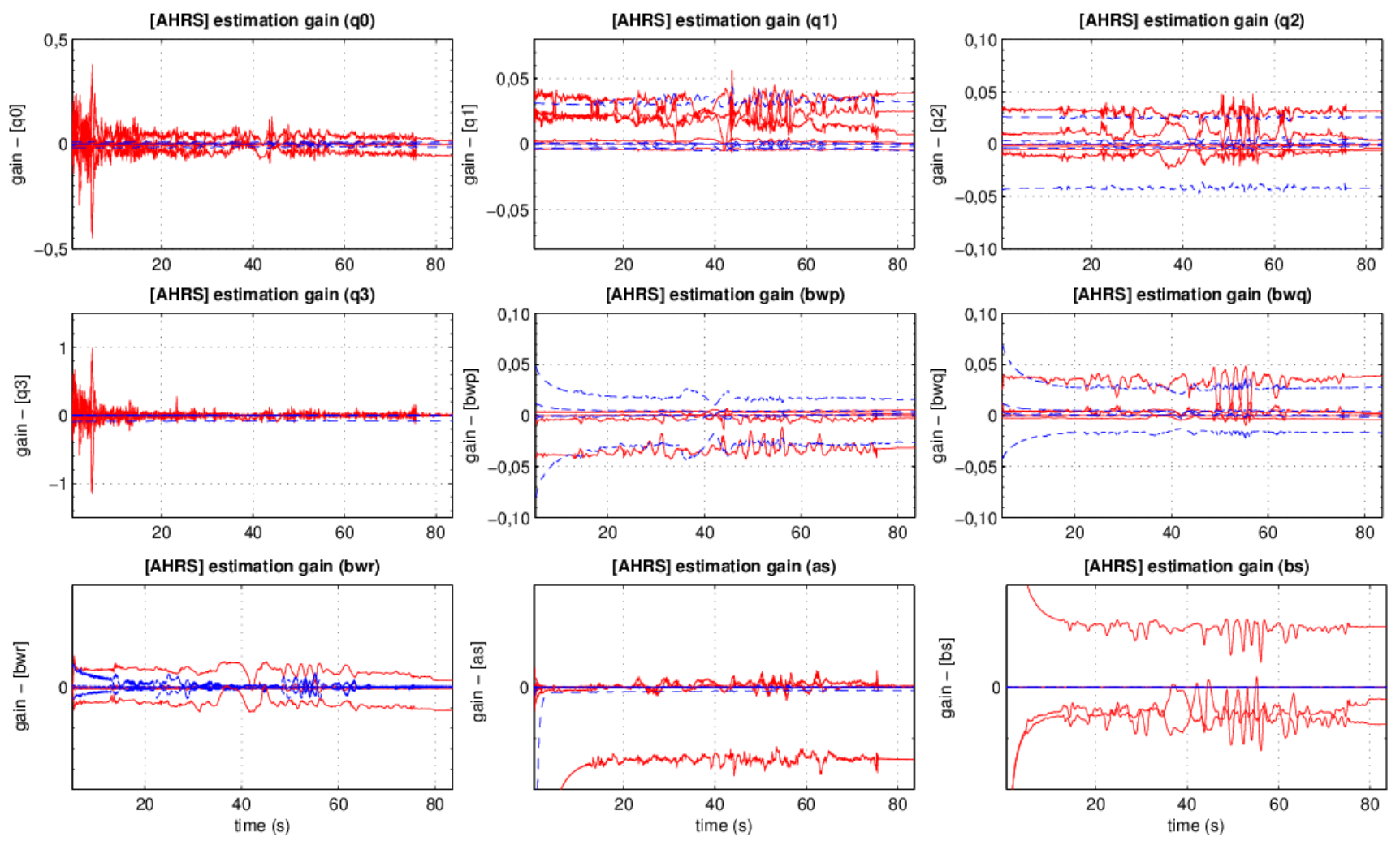

Fig. 6. Correction gains: comparison UKF (red) /IUKF (blue) (state components). 
brought by the invariant observer theory used to design our IUKF algorithm can be observed on Figures 5 and 6, which display, through time, both computed theoretical standard deviations and filters correction gains. By merging these results, it can be concluded that our proposed IUKF estimation technique is characterized by quasi-constant estimated standard deviations and correction gains w.r.t. any standard UKF estimation algorithm. Exploiting system's dynamics invariances in order to design nonlinear state estimation approaches allows to construct powerful nonlinear observers whose properties will be quasi-independent from the current followed trajectory.Therefore, state estimation uncertainties can be quantified by quasi-constant values through time (see for instance standard deviations on $\hat{\mathbf{q}}$ ).

This paves the way for designing less conservative, but robust, estimated state-feedback control laws in order to improve mini-UAVs flying and handling qualities. It is noteworthy that the reasoning made previously about the residual estimation uncertainties which impair $\left(\hat{\boldsymbol{\omega}}_{b}, \hat{a}_{s}, \hat{b}_{s}\right)$ for both techniques is still valid when analyzing the results of Figure 5. Although it has not been demonstrated by any theoretical proof, we can notice that, when they are consistent with UKF-based results (i.e., not for the state components related to gyrometer biases), the theoretical standard deviations computed by our proposed IUKF estimation technique minimize along the whole experiment the ones determined by the standard UKF methodology. This is especially remarkable for quaternion components estimates $\hat{\mathbf{q}}=\left(\begin{array}{llll}\hat{q}_{0} & \hat{q}_{1} & \hat{q}_{2} & \hat{q}_{3}\end{array}\right)^{T}$. Similarly to the theoretical standard deviations computed by the IUKF observer, the correction gains (cf. Fig. 6) appear less sensitive to the non-stationary noises levels, so that we can conclude that the invariant framework offers a better high frequency perturbations rejection in terms of filtering capabilities. In the case of the IUKF estimator, these gains could be also approximated by constant values, after a given transient regime, rather than in the case of the standard UKF algorithm.

\section{CONCLUSION AND FUTURE PROSPECTS}

This article has presented an innovative procedure to derive an invariant observer for nonlinear state estimation. This latter, named IUKF, combines both invariant observers theory and unscented filtering principles. The experimental results presented and gathered in $\S$ III-C have shown an equivalent capability of our proposed IUKF technique in comparison with an UKF method for nonlinear state estimation. These results have also permitted to check in realistic conditions some invariance properties which characterize our designed observer. Among these latter, stability of estimated standard deviations, which characterize estimated state trajectory uncertainties, must be highlighted since it could facilitate new control strategies design with less conservatism.

Future works will investigate the possibility to use constant gain matrices, optimized offline by the IUKF, into a complementary observer. The benefit of this solution would be to take advantage of the computational simplicity of the complementary observer but with optimal correction terms provided by the IUKF.

\section{REFERENCES}

[1] Aghannan, N. and al.(2002). On invariant asymptotic observers. In Proceedings of the 41st IEEE Conference on Decision and Control, Las Vegas (NV), USA, 10-13 December 2002 (pp. 1479-1484).

[2] Barczyk, M. and al. (2015). Invariant EKF Design for Scan MatchingAided Localization. IEEE Tansaction on Control Systems Technology, 23 (6), 2440-2448.

[3] Barczyk, M., \& Lynch, A. F. (2013). Invariant Observer Design for Helicopter UAV Aided Inertial Navigation System. IEEE Transaction on Control Systems Technology, 21 (3), 791-806.

[4] Barrau, A. and al. (2015). Intrinsic filtering on Lie groups with applications to attitude estimation. IEEE Tansaction on Automatic Control, 60 (2), 436-449.

[5] Barrau, A., \& Bonnabel, S. (2014). Invariant particle filtering with application to localization. In Proceedings of the 53rd IEEE Conference on Decision and Control, Los Angeles (CA), USA, 15-17 December 2014 (pp. 5599-5605).

[6] Barczyk, M.and al. (2015). Invariant EKF Design for Scan MatchingAided Localization. IEEE Tansaction on Control Systems Technology, 23 (6), 2440-2448.

[7] Bonnabel, S.and al. (2009). Non-linear symmetry-preserving observers on Lie groups. IEEE Tansaction on Automatic Control, 54 (7), 17091713.

[8] Bonnabel, S.and al. (2009). Invariant Extended Kalman Filter: Theory and Application to a Velocity-Aided Attitude Estimation Problem. In Proceedings of the 48th IEEE Conference on Decision and Control, Shanghai, China, 16-18 December 2009 (pp. 1297-1304).

[9] Bonnabel, S.and al.(2008). Symmetry-preserving observers. IEEE Tansaction on Automatic Control, 53 (11), 2514-2526.

[10] Condomines, J-P. and al. (2014). Pi-Invariant Unscented Kalman Filter for Sensor Fusion. In Proceedings of the 53rd IEEE Conference on Decision and Control, Los Angeles (CA), USA, 15-17 December 2014 (pp. 1035-1040).

[11] Condomines, J-P. and al. (2013). Nonlinear state estimation using an invariant unscented Kalman filter. In Proceedings of the 2013 AIAA Guidance, Navigation and Control Conference, Boston (MA), USA, 19-2 August 2013 (pp 1-15).

[12] Condomines, J.P. and al. (2017). Optimal Invariant Observers Theory for Nonlinear State Estimation. Multisensor Attitude Estimation Fundamental Concepts and Applications Edited by Fourati Hassen Belkhiat Djamel Eddine Chouaib Iniewski Krzysztof , Taylor \& Francis, pp 391-408, 2016, 978-1-4987-4571-0, ISBN: 978-1-4987-4580-2

[13] Crassidis, J. L. and al.(2007). Survey of Nonlinear Attitude Estimation Methods. Journal of Guidance, Control and Dynamics,30 (1), 12-28.

[14] Crassidis, J. L., and Junkins, J. L. (2004). Optimal Estimation of Dynamic Systems. Chapman \& Hall/CRC Press LLC.

[15] Diemer, S., and Bonnabel, S. (2015). An invariant linear quadratic Gaussian controller for a simplified car. In Proceedings of the 2015 IEEE Conference on Robotics and Automation, Seattle (WA), USA, 26-30 May 2015 (pp. 448-453).

[16] Julier, S. J.and al. (2004). Unscented filtering and nonlinear estimation. In Proceedings of the IEEE (Invited Paper), 92 (3), (pp. 401-422).

[17] Khosravian, A.and al. (2015). Observers for invariant systems on Lie groups with biased input measurements and homogeneous outputs. Automatica, 55 (Issue C), 19-26.

[18] Martin, P., \& Salaün, E. (2007). Invariant observers for attitude and heading estimation from low-cost inertial and magnetic sensors. In Proceedings of the 46th IEEE Conference on Decision and Control, New Orleans (LA), USA, 12-14 December 2007 (pp. 1039-1045).

[19] Sarkka, S. (2007). On unscented Kalman filtering for state estimation of continous-time nonlinear systems. IEEE Transaction on Automatic Control, 52 (9), 1631-1641.

[20] van der Merwe, R., \& Wan, E. A. (2001). The square-root unscented Kalman filter for state and parameter estimation. In Proceedings of the IEEE International Conference on Acoustics, Speech and Signal Processing, Salt Lake City (UT), USA, 7-11 May 2001 (pp. 34613464). 